\title{
Letter to the Editor regarding the paper by Aurilio et al., A meta-analysis of oestrogen receptor, progesterone receptor and human epidermal growth factor receptor 2 discordance between primary breast cancer and metastases
}

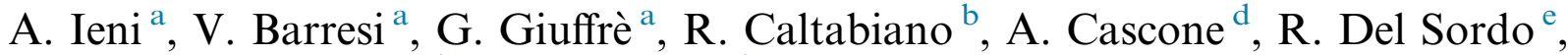 \\ D. Cabibi ${ }^{\mathrm{c}}$, P. Zeppa ${ }^{\mathrm{d}}$, S. Lanzafame ${ }^{\mathrm{b}}$, A. Sidoni ${ }^{\mathrm{e}}$, V. Franco ${ }^{\mathrm{c}}$, G. Tuccari ${ }^{\mathrm{a}, *}$
}

\footnotetext{
a Dipartimento di Patologia Umana "Gaetano Barresi", Università degli Studi di Messina, A. O.U. "Policlinico G. Martino", Messina, Italy

${ }^{\mathrm{b}}$ Dipartimento Anatomia, Patologia diagnostica, Medicina legale, Igiene e Sanità Pubblica, Università degli Studi di Catania, "Policlinico G. Rodolico", Catania, Italy

"Dipartimento di Patologia Umana, Università di degli Studi Palermo, A.O.U. "Policlinico Giaccone", Palermo, Italy

d Dipartimento di Medicina e Chirurgia, Azienda Ospedaliera Universitaria "San Giovanni di Dio e Ruggi d'Aragona", Università degli Studi di Salerno, Italy

'Dipartimento di Medicina Sperimentale e Scienze Biochimiche, Università degli Studi di Perugia, A.O.U. "S. Maria della Misericordia", Perugia, Italy
}

Sir

We have read with great interest the article in press of Aurilio et al., A meta-analysis of oestrogen receptor, progesterone receptor and human epidermal growth factor receptor 2 discordance between primary breast cancer and metastases (http://dx.doi.org/10.1016/j.ejca.2003. 10.004), which will appear in Eur J Cancer. In the paper, the Authors have performed a meta-analysis of the studies published in the literature concerning the discordance rate in oestrogen receptor (ER), progesterone receptor $(\mathrm{PgR})$ and HER2 status between primary tumour and corresponding relapse. It is well known that a considerable controversy concerns the issue of hormone receptors (HRs) expression as well as HER2 status between primary breast carcinomas (BC) and metastatic sites from the same patient, as elsewhere

\footnotetext{
* Corresponding author: Tel.: +39 90 2212539; fax: +39 902938150 . E-mail addresses: tuccari@unime.it, giovannituccari@yahoo.it (G. Tuccari).
}

reported in literature [1-5]. In particular, the discordance between primary $\mathrm{BC}$ and metastases from negative to positive and vice versa potentially affects the treatment regimen [5]; therefore, it has been suggested that neoplastic tissue of metastatic lesions should be systematically re-biopsied to optimally re-assess HR as well as HER2 [1,2,4-8].

In their meta-analysis, focusing on HER2 status, Aurilio et al. documented that the pooled discordance proportion with respect to the primary breast tumour was $10 \%$ for distant metastases and $6 \%$ for loco-regional relapses. Moreover, a different pooled discordant proportion was found on the basis of the different utilised technique, since it was $10 \%$ in studies using immunohistochemistry (IHC) and fluorescence in situ hybridisation (FISH), but $5 \%$ in studies using IHC only; therefore, they conclude the observed discrepancy was not merely due to technicality. Nevertheless, whether these changes in HER2 status may be attributed to laboratory reproducibility in techniques or to other pre-analytical fac- 
tors, such as not standardised inadequate fixation or, otherwise, to biological modifications and heterogeneity in tumours, still remains to be clarified. On the other hand, the hypothesis that variation in HER2 status may reflect clonal genome evolution has been stressed [7]; consequently, tumour heterogeneity may be attributable to tumour biological trend leading to clonal selection with the development of new clones, previously undetectable within the primary breast cancer. In any case, the significant discordance between HER2 status in the comparison of primary and recurrent/metastatic breast carcinomas may influence the correct treatment, determining an inappropriate use of targeted therapies [8-11].

In this intriguing field of breast pathology, we have started a retrospective multi-institution analysis to assess the discordance rate of HER2 status, not only in recurrent or distant metastatic breast lesions, but also taking into consideration the comparison between primary breast tumours and corresponding synchronous nodal metastases. For this purpose, 148 patients (mean age 59.27 years; range 33-96) not subjected to chemotherapy, were collected and retrospectively analysed from five italian anatomo-pathological units. Data concerning histotype, grading, hormone receptors expression, growth fraction (Ki67 LI) and HER2 status were largely available. Additionally, 14 metachronous distant recurrent breast lesions were also analysed. Patient personal details were non-identifiable and all patients had provided written consent to their medical information being used for research purposes, according with the Helsinki declaration.

For each case, $4 \mu \mathrm{m}$-thick sections from formalin-fixed paraffin-embedded tissue blocks were set for immunohistochemistry. In particular, the IHC analysis for HER2 status was done by automated workstation (DAKO Autostainer Link48) according to manufacturer's products and DAKO HercepTest ${ }^{\mathrm{TM}}$ kit; in each pathological unit, HER 2 positivity was defined as $3+$, when strong membranous staining in at least $30 \%$ cells was noted. FISH analysis was performed using HER2 FISH PharmDx ${ }^{\mathrm{TM}} \mathrm{kit}$ (Dako, Glostrup, Denmark) if previous IHC showed 2+ immunostaining (weak or moderate complete membranous staining of $10-30 \%$ of tumours cells); gene amplification was recorded when HER 2 to CEP17 signal ratio was $\geqslant 2.0$. All immunostained slides were reassessed by two observers (A.I. and G.T.), blinded of previous paired data, and the results were scored in a random order. The interobserver kappa $(\kappa)$ index in HER2 status determination was of 0.78 , representing a substantial agreement; when an inter-observer disagreement emerged, cases were jointly discussed until an agreement was reached. The Chi-square test was utilised to compare HER2 status with other biological parameters.

Table 1

Histopathological features and HER2 status changes in primary invasive ductal carcinomas and corresponding metastatic lymph nodes analysed.

\begin{tabular}{|c|c|c|c|c|c|c|}
\hline \multirow[t]{2}{*}{ Parameters } & \multicolumn{3}{|c|}{ Concordance $\mathrm{T} / \mathrm{N}$} & \multicolumn{3}{|c|}{ Discordance $\mathrm{T} / \mathrm{N}$} \\
\hline & All cases & $(+/+)$ & $(-/-)$ & All cases & $(+/-)$ & $\overline{(-/+)}$ \\
\hline HER2 status & 141 & 52 & 89 & 7 & 4 & 3 \\
\hline pT & 96 & 42 & 54 & 7 & 4 & 3 \\
\hline $\mathrm{T} 1$ & $37(94.9 \%)$ & 15 & 22 & $2(5.1 \%)$ & 1 & 1 \\
\hline $\mathrm{T} 2$ & $40(95.2 \%)$ & 18 & 22 & $3(7.0 \%)$ & 2 & 1 \\
\hline $\mathrm{T} 3$ & $10(90.9 \%)$ & 5 & 5 & $2(16.7 \%)$ & 1 & 1 \\
\hline $\mathrm{T} 4$ & $9(100 \%)$ & 4 & 5 & 0 & 0 & 0 \\
\hline $\mathrm{pN}$ & 97 & 42 & 55 & 7 & 4 & 3 \\
\hline $\mathrm{N} 1$ & $38(92.7 \%)$ & 17 & 21 & $3(7.3 \%)$ & 1 & 2 \\
\hline $\mathrm{N} 2$ & $39(92.9 \%)$ & 15 & 24 & $3(7.1 \%)$ & 2 & 1 \\
\hline N3 & $20(95.2 \%)$ & 10 & 10 & $1(4.8 \%)$ & 1 & 0 \\
\hline Grade & 110 & 44 & 66 & 7 & 4 & 3 \\
\hline G1 & $4(100 \%)$ & 0 & 4 & 0 & 0 & 0 \\
\hline G2 & $48(94.1 \%)$ & 14 & 34 & $3(5.9 \%)$ & 2 & 1 \\
\hline G3 & $58(93.5 \%)$ & 30 & 28 & $4(6.5 \%)$ & 2 & 2 \\
\hline Oestrogen receptor (ER) status & 111 & 44 & 67 & 7 & 4 & 3 \\
\hline $\mathrm{ER}+$ & $80(93.0 \%)$ & 22 & 58 & $6(7.0 \%)$ & 3 & 3 \\
\hline ER - & $31(96.9 \%)$ & 22 & 9 & $1(3.1 \%)$ & 1 & 0 \\
\hline Partial response (PR) status & 110 & 44 & 66 & 7 & 4 & 3 \\
\hline $\mathrm{PR}+$ & $73(92.4 \%)$ & 17 & 56 & $6(7.6 \%)$ & 3 & 3 \\
\hline PR - & $37(97.4 \%)$ & 27 & 10 & $1(2.6 \%)$ & 1 & 0 \\
\hline Ki67 & 111 & 44 & 67 & 6 & 4 & 2 \\
\hline KI $67 \leqslant 14 \%$ & $35(97.2 \%)$ & 7 & 28 & $1(2.8 \%)$ & 0 & 1 \\
\hline KI $67>14 \%$ & $76(93.8 \%)$ & 37 & 39 & $5(6.2 \%)$ & 4 & 1 \\
\hline
\end{tabular}


The analysed breast cancer casuistry is summarised in Table 1. The whole concordance rate for HER2 was 95.2\%; in detail, 89 cases were concordantly HER2 negative either in primary breast carcinoma or in node metastases, while 52 cases were HER2 positive in both samples. Changes in HER2 status between primary invasive ductal carcinomas and corresponding metastatic lymph nodes were found with a pooled discordant rate of $4.8 \%$. The discordance involved seven cases: three of which, primarily negative, became positive $(2.1 \%)$ while, by contrast, the original immunopositivity for HER2 disappeared in four cases (2.7\%). Moreover, a discordance rate was also appreciable in the comparative analysis between primary carcinomas and metachronous recurrences; in particular, $1 / 14$ cases $(7.14 \%)$ revealed a $3+$ immunoreactivity in secondary relapsed skin lesion, while the primary breast lesion was initially scored 0 .

No relationships emerged between HER2 status and hormone receptors, growth fraction and other clinicopathological findings (pT, pN, grade).

In conclusion, discordance in HER2 expression between primary and lymph node breast cancer seems to be ascertained by our multiple-institution analysis. The appearance of changes in HER2 status during the natural history of breast cancer may influence the clinical decision of oncologists; in fact, while the shift from positive to negative suggests to maintain the Trastuzumab as treatment choice, by contrast the opposite shift from negative to positive should be evaluated by a simultaneous HER2 determination in both primary and lymph node breast cancer lesions, influencing thus the targeted therapeutic protocol.

\section{Conflict of interest statement}

None declared.

\section{References}

[1] Aitken SJ, Thomas JS, Langdon SP, et al. Quantitative analysis of changes in ER, PR and HER2 expression in primary breast cancer and paired nodal metastases. Ann Oncol 2010;21(6): $1254-61$.

[2] Chen X, Yuan Y, Gu Z, et al. Accuracy of estrogen receptor, progesterone receptor, and HER2 status between core needle and open excision biopsy in breast cancer: a meta-analysis. Breast Cancer Res Treat 2012;134(3):957-67.

[3] Hoefnagel LD, Moelans CB, Meijer SL, et al. Prognostic value of estrogen receptor $\alpha$ and progesterone receptor conversion in distant breast cancer metastases. Cancer 2012;118(20):4929-35.

[4] Curtit E, Nerich V, Mansi L, et al. Discordances in estrogen receptor status, progesterone receptor status, and HER2 status between primary breast cancer and metastasis. Oncologist 2013;18(6):667-74.

[5] Penault-Llorca F, Coudry RA, Hanna WM, et al. Experts' opinion: recommendations for retesting breast cancer metastases for HER2 and hormone receptor status. Breast 2013;22(2):200-2.

[6] Idirisinghe PK, Thike AA, Cheok PY, et al. Hormone receptor and c-ERBB2 status in distant metastatic and locally recurrent breast cancer. Pathologic correlations and clinical significance. Am J Clin Pathol 2010;133(3):416-29.

[7] Aurilio G, Disalvatore D, Pruneri G, et al. A meta-analysis of oestrogen receptor, progesterone receptor and human epidermal growth factor receptor 2 discordance between primary breast cancer and metastases. Eur J Cancer 2013. http://dx.doi.org/ 10.1016/i.ejca.2003.10.004.

[8] Pérez-Fidalgo JA, Eroles P, Ferrer J, et al. An evaluation of the impact of technical bias on the concordance rate between primary and recurrent tumors in breast cancer. Breast 2013;22(5):974-9.

[9] Houssami N, Macaskill P, Balleine RL, et al. HER2 discordance between primary breast cancer and its paired metastasis: tumor biology or test artefact? Insights through meta-analysis. Breast Cancer Res Treat 2011;129(3):659-74.

[10] Cardoso F, Harbeck N, Fallowfield L, et al. Locally recurrent or metastatic breast cancer: ESMO Clinical Practice Guidelines for diagnosis, treatment and follow-up. Ann Oncol 2012;23(Suppl. 7):vii11-9.

[11] Turner NH, Di Leo A. HER2 discordance between primary and metastatic breast cancer: assessing the clinical impact. Cancer Treat Rev 2013;39(8):947-57. 